CERN-PPE/95-98

July 5,1995

\title{
A 1024 PAD SILICON DETECTOR TO SOLVE TRACKING AMBIGUITIES IN HIGH MULTIPLICITY EVENTS
}

\author{
S. Simone, M.G. Catanesi, D. Di Bari, V. Didonna, D.Elia, \\ B. Ghidini, V. Lenti, V. Manzari and E. Nappi \\ Dipartimento di Fisica dell'Università and Sezione INFN, Bari
}

\begin{abstract}
Silicon detectors with two-dimensional pad readout have been designed and constructed for the WA97 experiment at CERN, in order to solve ambiguities for track reconstruction in a silicon microstrip telescope. A high density fan out has been developed on a glass support to allow the electrical contacts between the detector and the front end electronics.

Silicon pad detectors have been successfully operated both during the proton- $\mathrm{Pb}$ and $\mathrm{Pb}-\mathrm{Pb}$ runs of the WA97 experiment.
\end{abstract}

Submitted to Nuclear Instruments and Methods in Physics Research A 
The WA97 experiment at the CERN Super Proton Synchrotron (SPS) has been designed to measure the spectra of hyperons and antihyperons produced in ultrarelativistic lead-lead interactions in the central rapidity region with medium to high transverse momentum[1]. The ultimate goal of such experiment is to find evidence for a new state of matter, the Quark Gluon Plasma (QGP), which is predicted[2] to occur in head-on collisions of heavy ions because of the large energy and baryon densities obtainable over a relatively large volume.

To handle the high multiplicity events expected in lead-lead collisions at $160 \mathrm{Gev} / \mathrm{c}$ per nucleon and to reconstruct the hyperon decay vertices, a telescope consisting of 18 silicon detector planes was developed. Detectors were arranged into 6 triplets each composed by 2 microstrip and 1 bidimensional pad detectors (fig. 1 ).

The $50 \mu \mathrm{m}$ pitch microstrip detectors were intended to determine the position of the incoming tracks with very high accuracy, while the bidimensional pad detectors would solve the ambiguities in the high multiplicity track environment.

\section{$2 \quad$ Pad detector description}

The $5.12 \times 5.12 \mathrm{~cm}^{2}$ detector sensitive area is segmented into 1024 pads, grouped as a $32 \times 32$ diode matrix.

The pad size ranges from $1.01 \times 1.60 \mathrm{~mm}^{2}$ to $2.38 \times 1.60 \mathrm{~mm}^{2}$ (fig.2). The segmentation of the detector has been chosen to get costant occupancy for all pads. Montecarlo simulations[1] show that the pseudorapidity density distribution of charged particles is nearly constant in the range of telescope acceptance $(3.0 \leq \eta \leq 3.9)$, resulting in the fact that all pad rows should cover the same pseudorapidity interval $(\Delta \eta=0.027)$. The pad size minimizes the probability to have in the same event more particles crossing a single pad so optimizing the efficiency of the detector in solving ambiguities. The maximum occupancy estimated by the Montecarlo is about $2 \%$.

Detectors have been manufactured by Eurisys Mesures (formerly IntertechniqueNucleaire) on a $10 \mathrm{~K} \Omega \mathrm{cm}$ n-type silicon bulk $300 \mu \mathrm{m}$ thick. The ion-implanted diode structures consist of p-type regions separated by $30 \mu \mathrm{m}$ of oxide and each covered by a $0.4 \mu \mathrm{m}$ thick Al layer for the ohmic contacts.

A $30 \mu \mathrm{m}$ oxide separates peripheric diodes from a guard ring which consists of a 50 $\mu \mathrm{m}$ wide p-type implanted strip, surrounding the detector sensitive area, the purpose of which is to reduce the leakage current.

The fan out of the detector has been realized on a slotted glass layer $800 \mu \mathrm{m}$ thick, with a surface $15 \times 15 \mathrm{~cm}^{2}$. As shown in fig.3, 8 kapton connectors (128 channels each) placed on the glass support and bounded on the golden fan out, provide the connection with the preamplifier electronics.

The glass was glued on the detector superimposing the segmented face; 16 slots, obtained by laser cut and partially uncovering all 1024 diodes, allow the wire bondings between the diode pads and the golden fan out. The golden fan out on the glass, due to the high density of pads, requests leads down to $8 \mu \mathrm{m}$ width and $14 \mu \mathrm{m}$ spaced, while 
the bonding pads close to each diode are $50 \mu \mathrm{m}$ large and $300 \mu \mathrm{m}$ long. The glass golden fan out was manufactured by means of a photolitographic process. The mask production required the use of an electron beam due to the narrow lead pitch in the most critical regions localized in between the slots. The golden leads, $4 \mu \mathrm{m}$ thick, were obtained with a galvanic growing starting from a $1 \mu \mathrm{m}$ thick cromium substrate. The region with higher leads density were localized among the 16 slots. The slot width ranges from $0.9 \mathrm{~mm}$ to 2 $\mathrm{mm}$. The spacing among the slots changes, due to the increasing dimension of the pads, from $1 \mathrm{~mm}$ to $2 \mathrm{~mm}$. The laser cut was realized by a $\mathrm{CO}_{2}$ pulsed Laser.

The use of glass for the support was requested to get a flatness around $0.1 \mu \mathrm{m}$, necessary for a $8 \mu \mathrm{m}$ wide leads, and a low thermical conductivity to avoid the melting of the bonding pads ( $200 \mu \mathrm{m}$ close to the laser cut point) which occurred using ceramic supports[3]. On the other hand, it was not possible to lower glass support thickness down to $800 \mu \mathrm{m}$, due to the occurrence of cracks during the laser cut.

\section{$3 \quad$ Pad detector performance}

A detector prototype has been tested in laboratory: the readout electronics used for the test consisted of an AMPLEX amplifier[4] (16 multiplexed channels per chip), followed by a DRAMS card[5] with an 8-bit flash ADC which provides a conversion with a $1 \mathrm{MHz}$ clock. An electrical pulse has been used to inject a charge to the input of the preamplifiers. A linear calibration curve was found when the injected charge is around $4 \mathrm{fC}$, corresponding to the most probable value for a minimum ionizing particle (MIP). These measurements have been used to calibrate the different gains for all AMPLEX channels.

The detector noise is reported in fig.4, where the pedestal r.m.s. distributions, corresponding to the situation with and without the detector connected to the electronics, are shown. The average noise is around 1300 electrons, of which about 900 electrons is the contribution of the readout electronics. Also a small fraction of very noisy pads, having noise greater than 2000 electrons, have been found: these channels have been suppressed in the following analysis. The difference of about 400 r.m.s. electrons between the noise with the detector connected and the one due to electronics contribution, is consistent with the AMPLEX specifications[4], where 33 r.m.s. electrons per pF of input capacitance is expected. In fact the load capacitance measured at the level of fan out, shown in fig.5 as a function of the detector depletion voltage, is about $13 \mathrm{pF}$ at full depletion.

A ${ }^{90} \mathrm{Sr}$ beta source and a trigger consisting of a single scintillator have been used to perform measurements about signal-to-noise ratio, charge sharing and capacitive cross talk effects.

Fig.6 shows the pulse hight distribution for events having a single hit pad, after pedestal subtraction and assuming a $4 \sigma$ cut in the hit selection ( $\sigma$ being the r.m.s. of the pedestal distribution for each channel). The most probable energy loss corresponds to a charge of about $3.8 \mathrm{fC}$, corresponding to the typical value expected for a minimun ionizing particle crossing a $300 \mu \mathrm{m}$ thick silicon detector[6].

The yield of not working channels, i.e. channels for which the pulse height is over the threshold only for a few percent of total events, turned out to be about $4 \%$ of the 
total, randomly distributed on the detector. The distribution of the signal to noise ratio for all pads is shown in fig.7, leading to an average value around 18 and with just a small fraction of noisy pads at lower values.

In order to study charge sharing between neighbouring pads, a cluster finding alghoritm has been used: a cluster is defined as a single hit pad or more ones but having a common side. In fig. 8 is shown the distribution of the cluster size (i.e. the number of pads per cluster): as can be seen, the single pad clusters largely dominate, being about $13 \%$ the fraction of clusters involving more pads.

Events having a single cluster with size two have been studied (about 7000, i.e. 10\% of the total). This kind of events could be originated by a particle crossing the detector near the pad boundary, so that the ionization charge is shared between the neighbours. In fig. 9 is reported the scatter plot of the pulse heights recorded in the two-pad clusters: for a large fraction of events (those in the lower left region) the total charge of the cluster is around 1 MIP, corresponding to the expected charge sharing effect.

In order to evaluate this charge sharing, on a sample of about 10000 events, we calculated the fraction of events having two neighbouring pads with pulse heights greater than $4 \sigma$ cut and a total cluster charge less than $6 \mathrm{fC}$ (which corresponds to a charge exceeding by about two standard deviations the typical MIP value). The occurrence of such events was found around $1 \%$ of the total.

Finally we have looked for capacitive cross talk effects: they can arise from particles crossing a pad anywhere in its area, with an opposite signal induced to a neighbouring due to capacitive coupling between the two fan out leads. The interest to verify this kind of effect is due to the particular fan out used, in which the golden leads follow a long path being very close to each other.

For this pourpose, pairs of pads whose fanout leads are very close (about $14 \mu \mathrm{m}$, in the lower part of the detector) have been examinated: fig.10a shows the distribution of the charge detected in a pad when one of the two pads having adiacent lead was hit. The negative values of the pulse height are compatible with the pedestal fluctuations, as shown in fig.10b; than there is no evidence for cross talk effects. Of course, the tail at positive values is due to charge sharing.

Two of these detectors have been operated during the first proton- $\mathrm{Pb}$ and $\mathrm{Pb}$ $\mathrm{Pb}$ runs of WA97 experiment at CERN-SPS, using a digital readout like that used for microstrip detectors in the silicon telescope[7]. They were placed in the last two triplets of the silicon telescope, about $150 \mathrm{~cm}$ and $170 \mathrm{~cm}$ downstream of the target respectively. Fig.11 shows the distribution of hit per event for one of the two detectors, during the ion run. The corresponding hit map for about $10000 \mathrm{~Pb}-\mathrm{Pb}$ events is reported in fig.12: as can be seen, the number of dead or very noisy channels is only a few percent of the total.

About 10000 straight beam tracks recorded during the proton run were analyzed and the correlation between the hit positions on the two detectors is shown in fig.13. The distributions of the difference in $y$ and in z between the hit channels on each plane show a clear central peak with negligible background due to noisy pads or tracks crossing with nonzero angle with respect to the perpendicular. 
Silicon pad detectors with two-dimensional readout have been built and tested: they are used in WA97 experiment at CERN, together with microstrips detectors, in order to handle the high multiplicity environment of ultrarelativistic $\mathrm{Pb}-\mathrm{Pb}$ collisions.

Results from laboratory tests show that the most probable energy loss corresponds to a charge of about $4 \mathrm{fC}$, as expected for a minimum ionizing particle. The signal to noise ratio has been found around 18 . The occurrence of charge sharing effects has been found to be of a few percents, while there is no evidence for capacitive cross talk effects between the fan out leads.

Two of these detectors have been successfully operated during the proton and ion runs of the WA97 experiment at the CERN-SPS.

\section{Acknowledgements}

We are specially grateful to INFN of Genova for the use of the $\mathrm{CO}_{2}$ laser and to R. Cereseto that realized the laser cut of the glass support.

We acknowledge also L. Liberti for his contribution in the preparation of kapton fan out.

\section{References}

[1] WA97 Proposal, CERN/SPSLC/91-29 (1991)

[2] J. Rafelski and B. Müller, Phys. Rev. Lett. 48 (1982) 1066

[3] P.W. Atkins, Physical Chemistry, Oxford University Press, 1988.

[4] E. Beuville et al., NIM A288 (1990) 157-167

[5] E. Chesi et al., DRAMS: a digital read out for multiplexed systems, Internal Note CERN/JETSET/EP (1988)

[6] R.H. Beuttenmuller et al., NIM A253 (1987) 500-510

[7] A. Beer et al., IEEE Trans. Nucl. Sci. 39 (1992) 546-550 


\section{Figure caption}

Fig.1 Layout for silicon telescope in WA97.

Fig.2 Pad detector matrix dimensions $(\mathrm{mm})$.

Fig.3 Support layer and kapton fan out for pad detector.

Fig.4 Noise distribution measured at the AMPLEX output.

Fig.5 Load capacitance measured at the fan out as a function of applied voltage.

Fig.6 Pulse height spectrum for events collected using a beta source.

Fig.7 Signal to noise distribution for all pads.

Fig.8 Cluster lenght distribution.

Fig.9 Scatter plot of the two charges recorded in 2-pad cluster events.

Fig.10 (a) Charge recorded in pads whose fan out is adiacent to the one of an hit pad (b) pedestal fluctuations for all pads.

Fig.11 Distribution of hit per event for pad detector in WA97 $\mathrm{Pb}-\mathrm{Pb}$ run

Fig.12 Pad detector hit map in $\mathrm{Pb}-\mathrm{Pb}$ events.

Fig.13 Y and Z correlations between pad detectors in the proton test beam, in units of pad dimensions. 


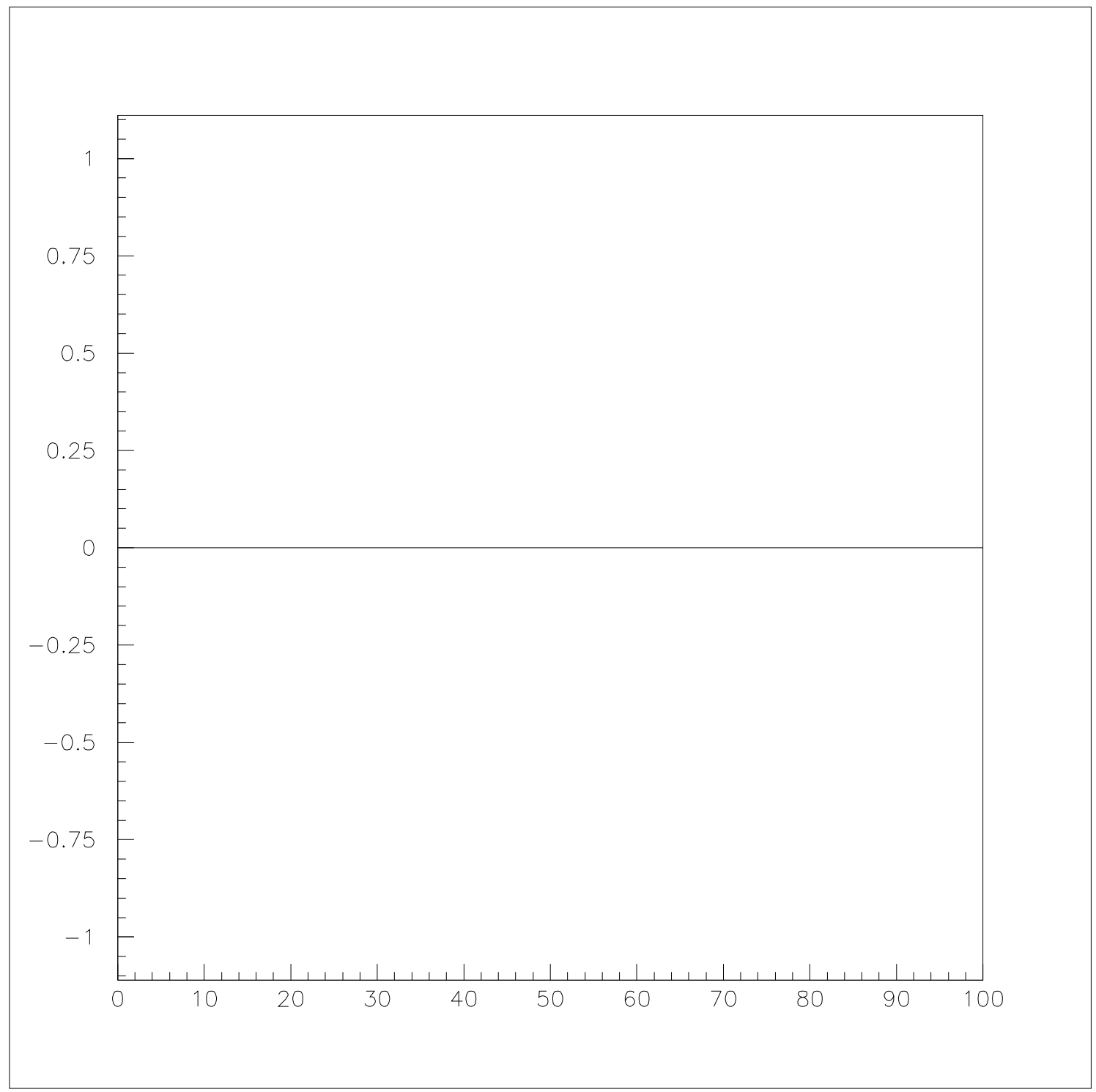

Fig. 1 


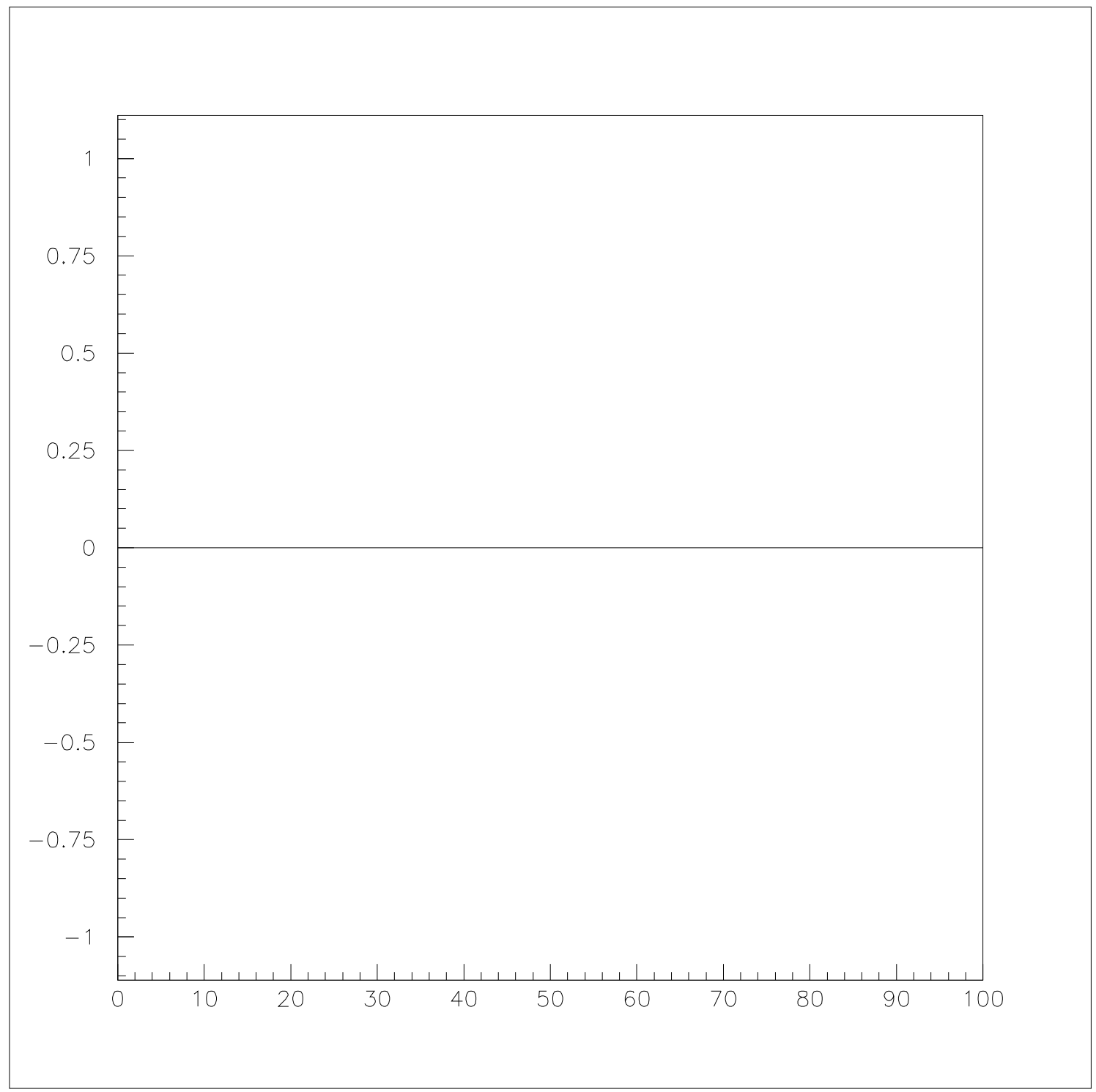

Fig. 2 


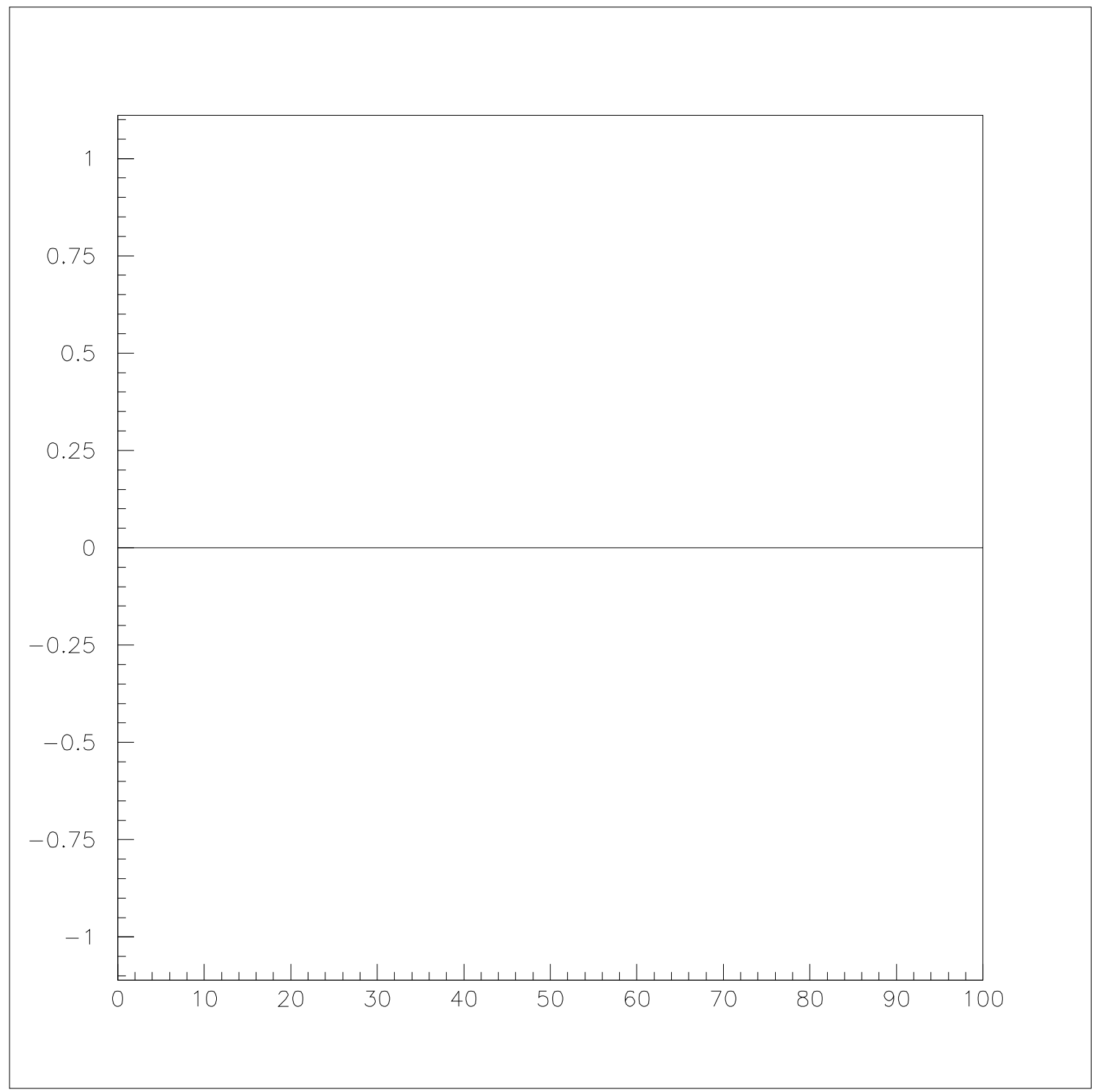

Fig. 3 


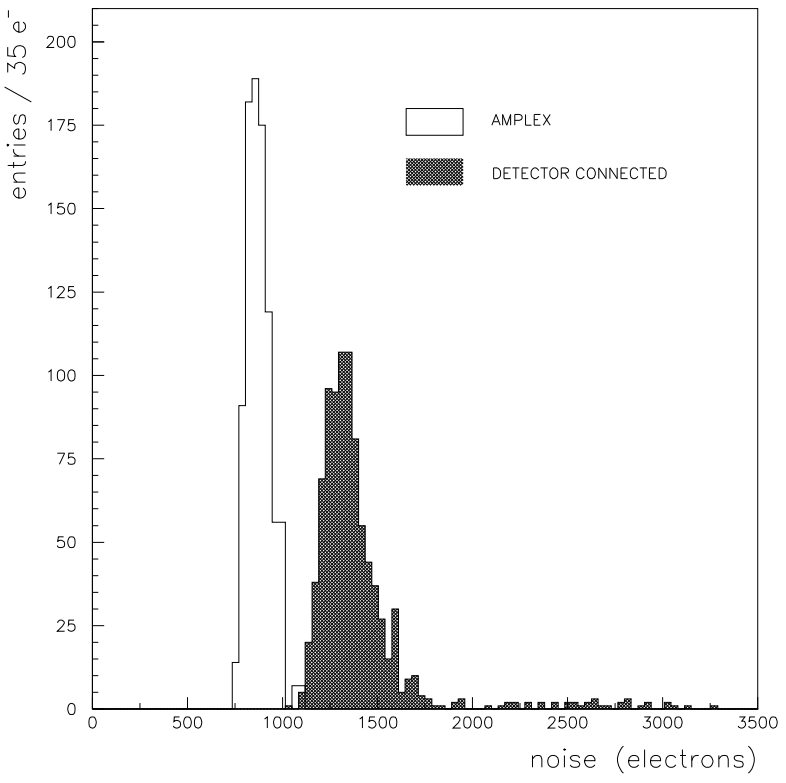

Fig. 4

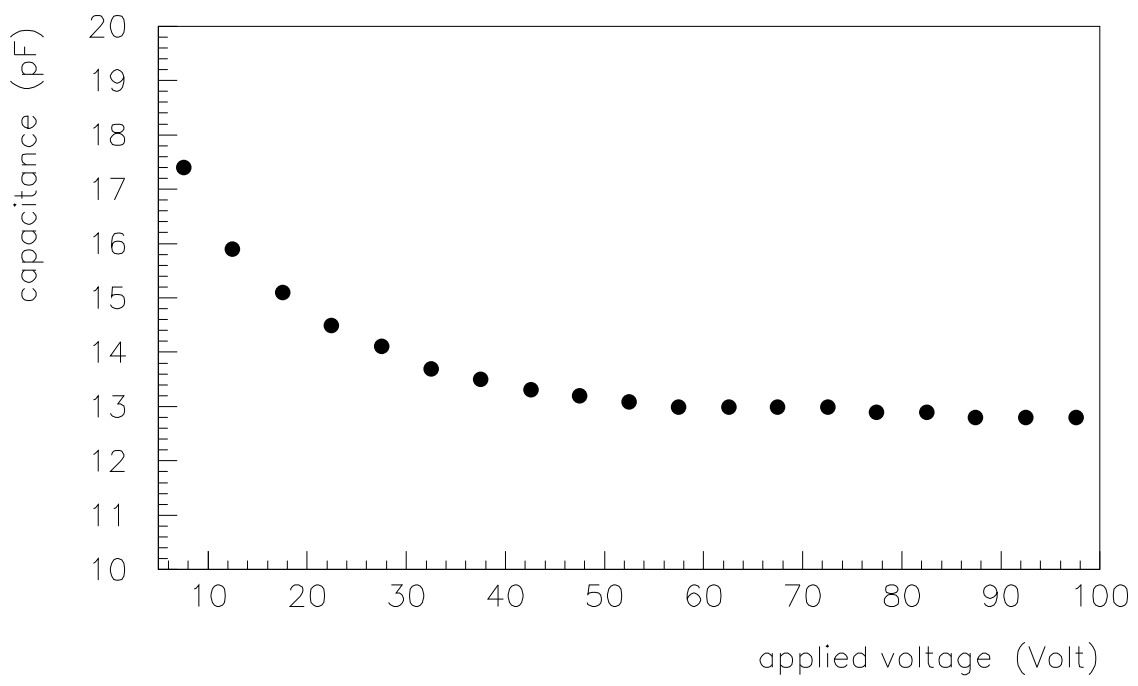

Fig. 5 


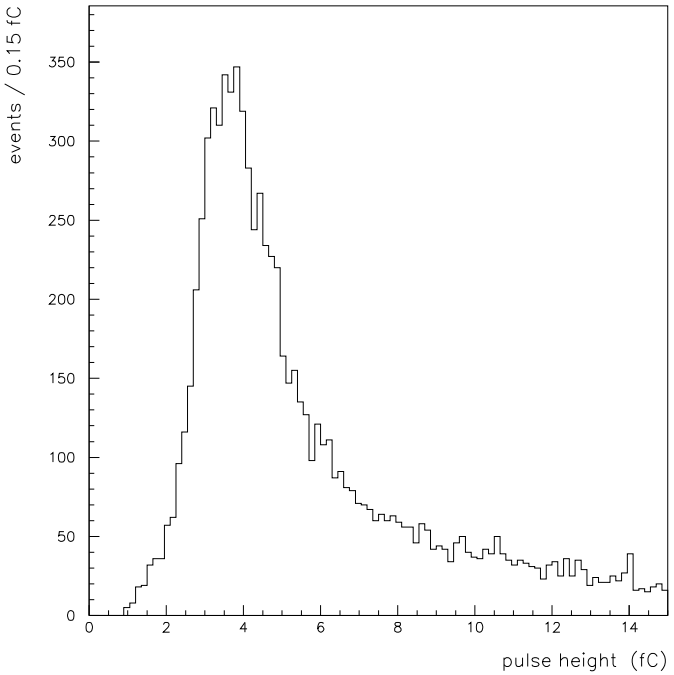

Fig. 6

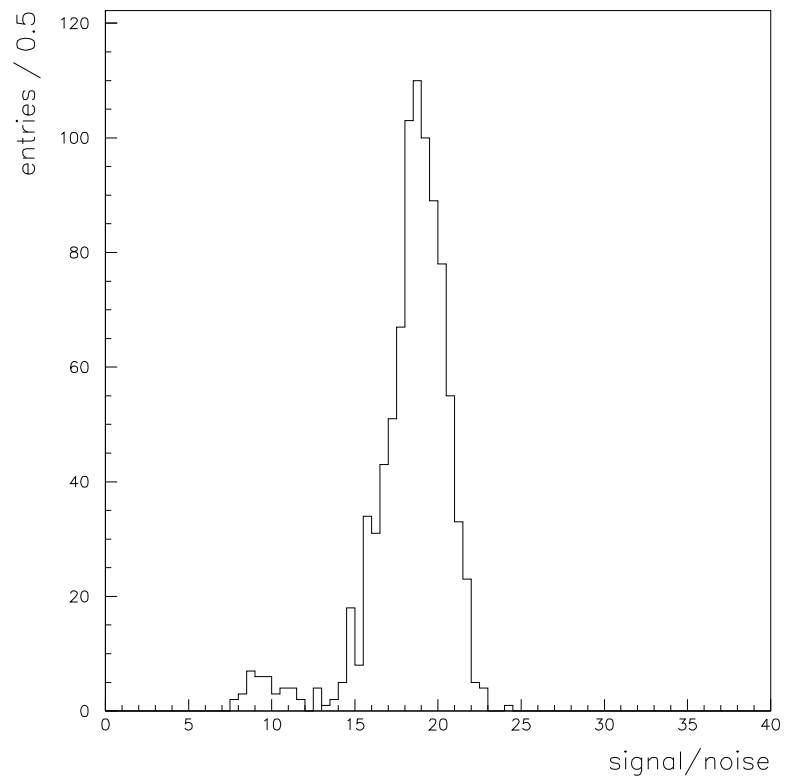

Fig. 8 


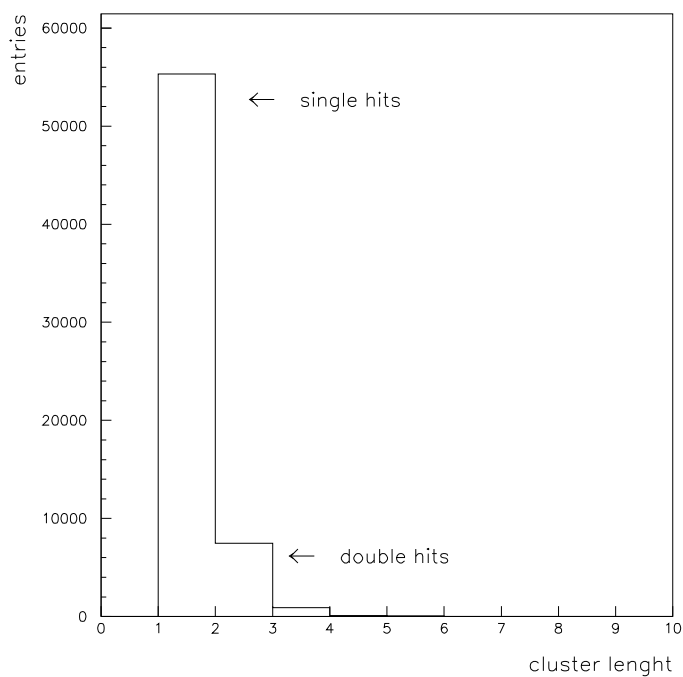

Fig. 8

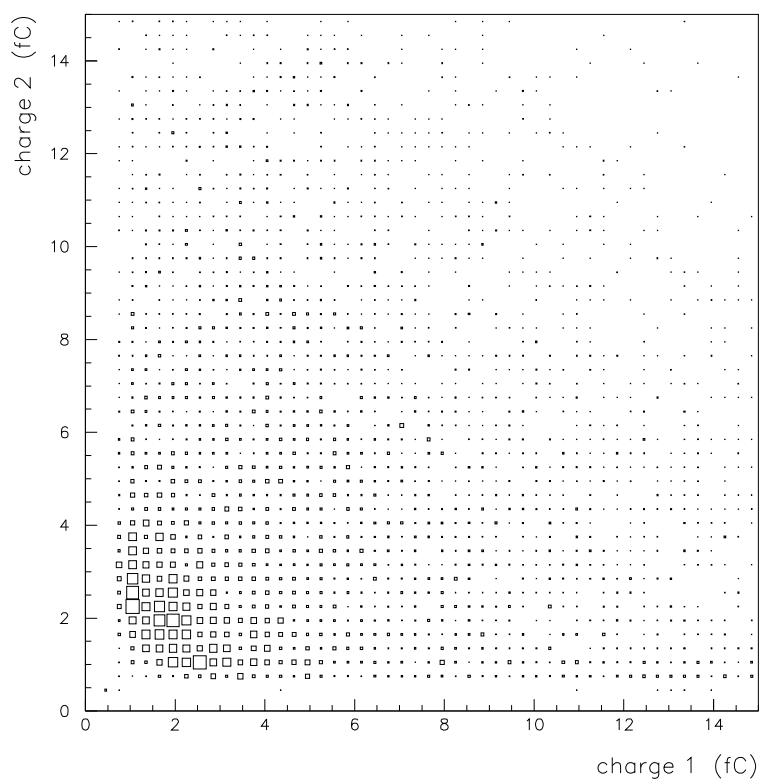

Fig. 9 

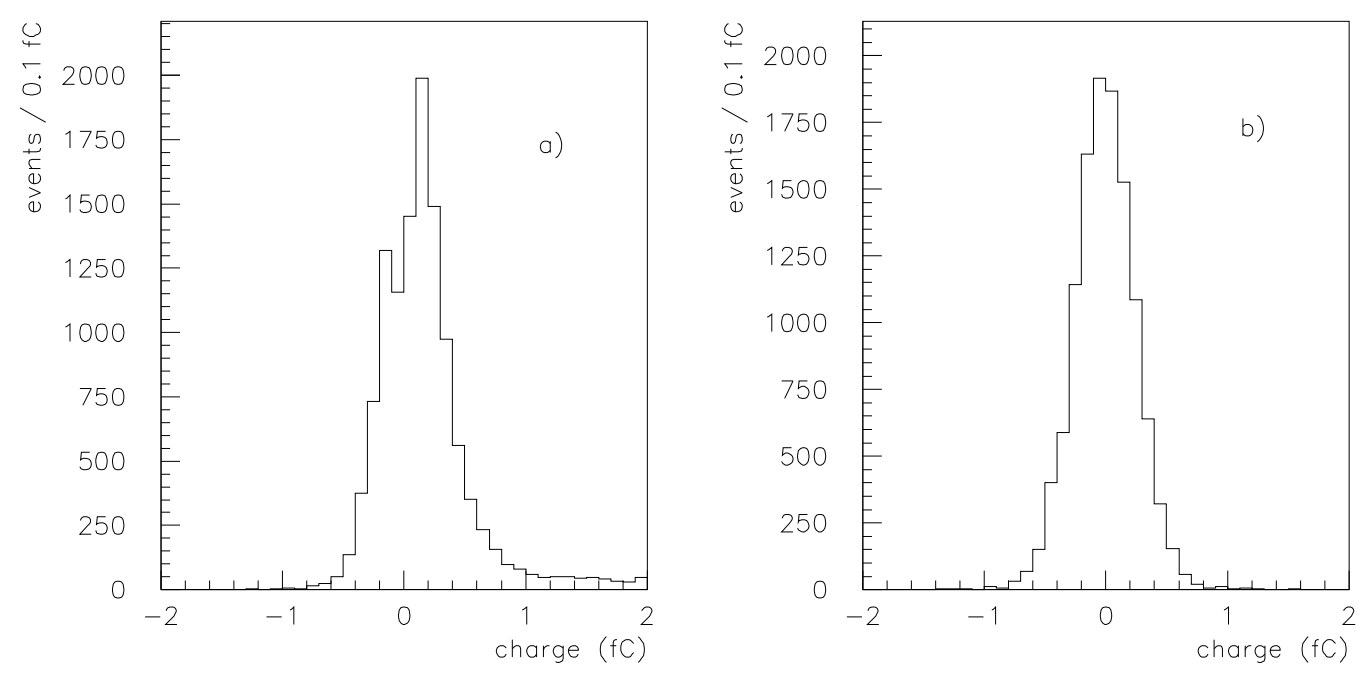

Fig. 10

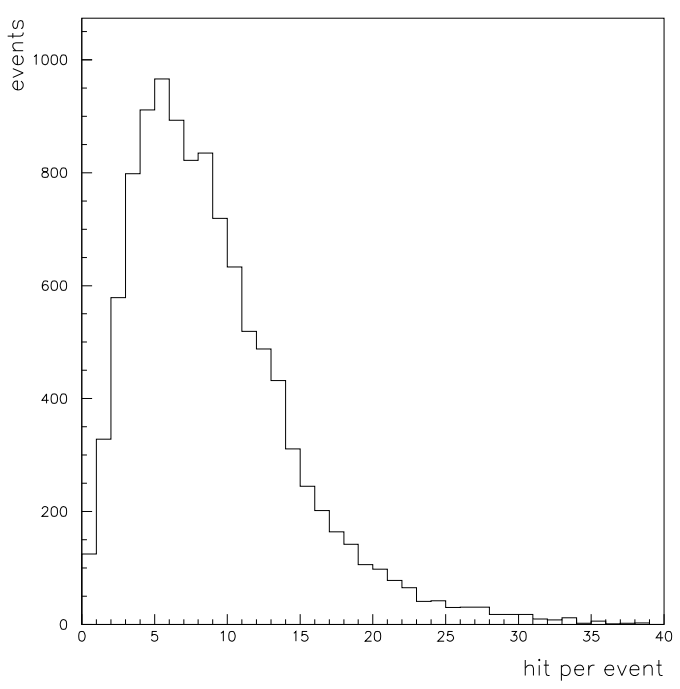

Fig. 11 


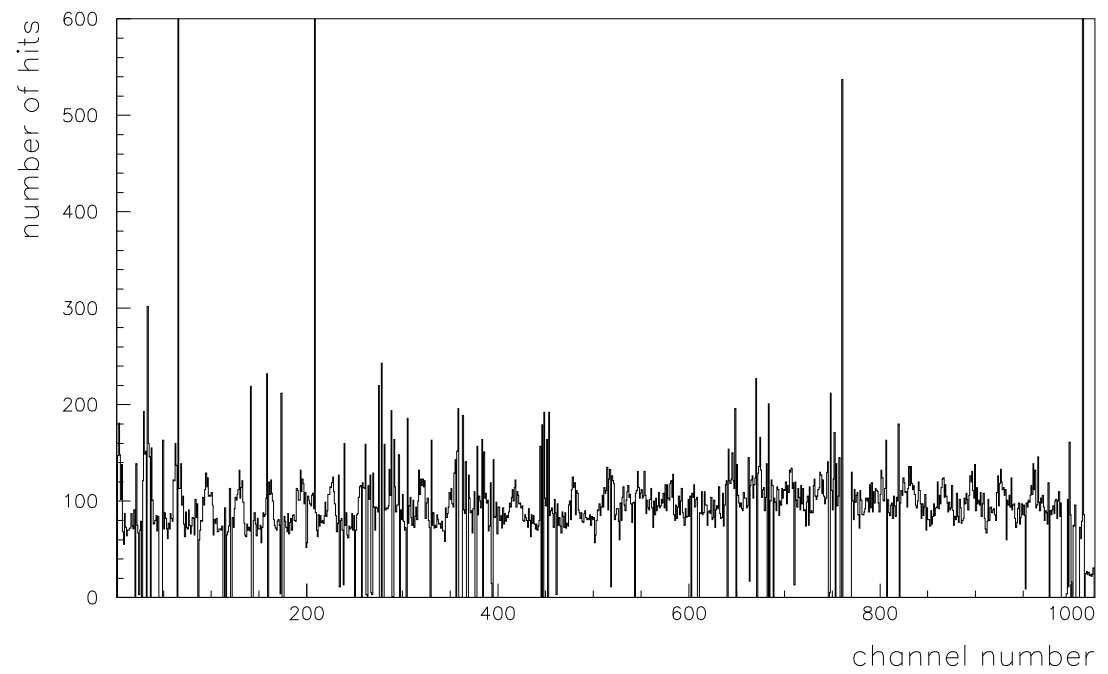

Fig. 12
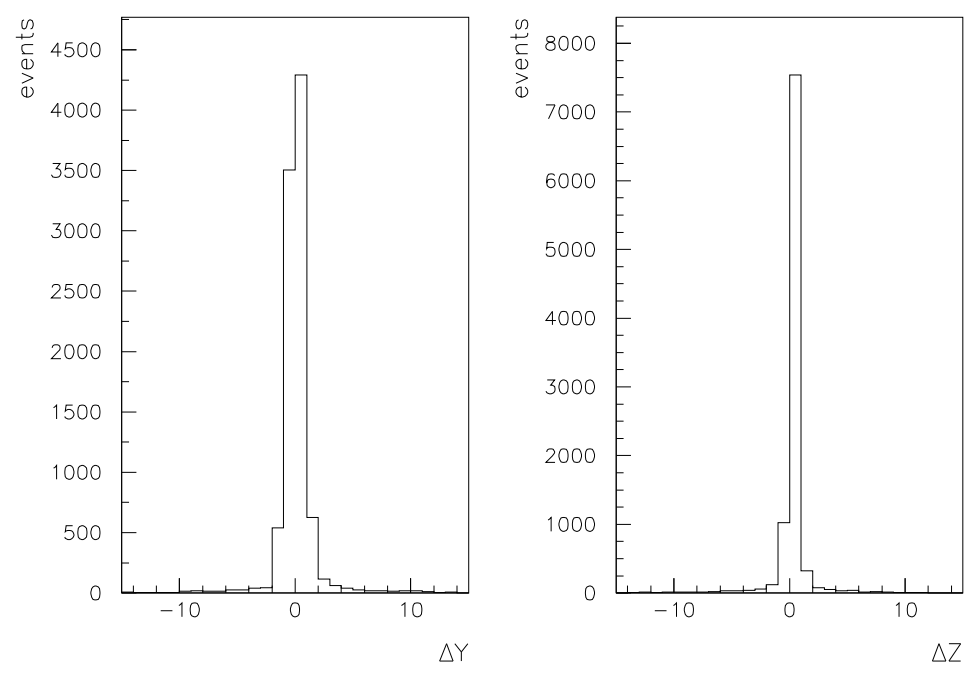

Fig. 13 